CUBO A Mathematical Journal

Vol.17, No 03, (71-90). October 2015

\title{
Computing the Laplace transform and the convolution for more functions adjoined
}

\author{
TAKAHIRO Sudo \\ Department of Mathematical Sciences, \\ Faculty of Science, University of the Ryukyus, \\ Senbaru 1, Nishihara, Okinawa 903-0213, Japan. \\ sudo@math.u-ryukyu.ac.jp
}

\begin{abstract}
We compute explicitly the Laplace transform and the convolution for more functions on the real than the continuous functions that obtained as the inverse Laplace transforms of rational functions on the complex plane vanishing at infinity. We also consider the algebraic structure with more functions adjoined.
\end{abstract}

\section{RESUMEN}

Calculamos explícitamente la transformada de Laplace y la convolución para más funciones en los reales que las funciones continuas obtenidas como transformadas de Laplace inversas de funciones racionales en el plano complejo que se anulan en el infinito. También consideramos la estructura algebraica con más funciones adjuntadas.

Keywords and Phrases: Laplace tranform, Gaussian function, Dirac delta function, convolution. 2010 AMS Mathematics Subject Classification: 44A10, 44A35, 26C15, 26A06, 26 A09. 


\section{Introduction}

This paper is a continuation from the paper [3] of the author, in which we compute explicitly the inverse Laplace transform for rational functions on the complex plane vanishing at infinity and consider the algebraic structure for the corresponding algebra of continuous elementary functions on the real $\mathbb{R}$ for which the Laplace transform on the infinite interval $[0, \infty)$ is defined.

In this paper, we compute explicitly the Laplace transform for more functions on the real than the continuous functions that obtained as the inverse Laplace transforms of rational functions on the complex plane vanishing at infinity. For this, we compute the convolution product with more functions adjoined, such as Gaussian functions, the Dirac delta functions, and the shifted delta functions. We also consider the algebraic structure with more functions adjoined.

This paper after this introduction is organized as follows: 2 Adjoining Gaussian functions; 3 Algebraic structure with Gaussian functions; 4 Adjoining the delta function; 5 Algebraic structure with the delta function; 6 Adjoining the shifted delta functions; 7 Algebraic structure with the shifted delta functions; 8 More with the weak derivative.

Our elementary explicit computation results obtained and algebraic consideration results obtained should be useful as a reference.

Refer to [4] or [2] (or [1]) for some basics about the Laplace transform.

Notation. We denote by $A(\mathbb{R})$ the real algebra with convolution, generated by the three sets of elementary continuous functions on the real line $\mathbb{R}$ with $t$ real variable: $\left\{t^{n} \mid n \in \mathbb{N}=\{0,1,2, \cdots\}\right\}$ of monomials and $\left\{e^{\mu t} \mid \mu \in \mathbb{R}\right\}$ of exponential functions based to $e$ and $\{\sin \lambda t, \cos \lambda t \mid \lambda \in \mathbb{R}\}$ of trigonometric functions and their point-wise multiples such as $t^{n} e^{\mu t} \sin \lambda t$.

The (extended in our sense) convolution product for $A(\mathbb{R})$ (but the same as the usual one by restriction to $[0, \infty))$ is defined as the (Riemann) integral:

$$
f * g=(f * g)(t)=\int_{0}^{t} f(t-\tau) g(\tau) d \tau, \quad(t \in \mathbb{R})
$$

for $f, g \in A(\mathbb{R})$, which makes sense and is a commutative and associative operation as well known as the usual case on the interval $[0, \infty)$.

Denote by $\mathbb{C}$ the complex plane.

For $f=f(t)$ a suitable function on $\mathbb{R}$ (as in $A(\mathbb{R})$ ), the (extended in our sense) Laplace transform of $f$ (but the same as the usual one of $f$ restricted to $[0, \infty)$ ) is defined by the (Riemann) integral:

$$
\mathrm{L}(\mathbf{f}(\mathrm{t}))=\mathrm{L}(\mathbf{f}(\mathrm{t}))(\mathrm{s})=\int_{0}^{\infty} e^{-s t} \mathbf{f}(\mathrm{t}) \mathrm{dt}
$$

for $s \in \mathbb{C}$ (formally or in the domain of convergence). 


\section{Adjoining Gaussian functions}

Quite well known, but first of all we consider Gaussian functions as follows:

Lemma 2.1. For $t \in \mathbb{R}, s \in \mathbb{C}$ and $\lambda \in \mathbb{R}$ with $\lambda>0$, we have

$$
\mathrm{L}\left(e^{-\lambda \mathrm{t}^{2}}\right)=\frac{\sqrt{\pi}}{2 \sqrt{\lambda}} e^{\frac{\mathrm{s}^{2}}{4 \lambda}},
$$

Proof. Check that

$$
e^{-s t} e^{-\lambda t^{2}}=e^{-\lambda\left(t+\frac{s}{2 \lambda}\right)^{2}+\frac{s^{2}}{4 \lambda}}
$$

and then

$$
L\left(e^{-\lambda t^{2}}\right)=e^{\frac{s^{2}}{4 \lambda}} \int_{0}^{\infty} e^{-\lambda\left(t+\frac{s}{2 \lambda}\right)^{2}} d t .
$$

Using change of variables and Cauchy's theorem, we obtain that the integral is computed to be equal to

$$
\begin{aligned}
& \left.\int_{\frac{s}{2 \sqrt{\lambda}}+0}^{\frac{s}{2 \sqrt{\lambda}}+\infty} e^{-u^{2}} \frac{d u}{\sqrt{\lambda}} \text { with } u=\sqrt{\lambda}\left(t+\frac{s}{2 \lambda}\right)\right) \\
& =\frac{1}{\sqrt{\lambda}} \int_{0}^{\infty} e^{-u^{2}} d u=\frac{\sqrt{\pi}}{2 \sqrt{\lambda}} .
\end{aligned}
$$

Lemma 2.2. For $t \in \mathbb{R}, s \in \mathbb{C}$ and $\lambda, \mu \in \mathbb{R}$ positive, we have

$$
\mathrm{L}\left(e^{-\lambda \mathrm{t}^{2}} * e^{-\mu \mathrm{t}^{2}}\right)=\frac{\pi}{4 \sqrt{\lambda \mu}} e^{\frac{\lambda+\mu}{4 \lambda \mu} s^{2}} .
$$

We need to check the convolution explicitly as follows.

Proposition 1. For $t \in \mathbb{R}$ and positive $\lambda, v \in \mathbb{R}$,

$$
\begin{aligned}
& \left(e^{-\lambda t^{2}} * e^{-\mu t^{2}}\right)(t)=\frac{1}{\sqrt{\lambda+\mu}} e^{-\lambda\left(1-\frac{\lambda}{\lambda+\mu}\right) t^{2}} \int_{\frac{-\lambda t}{\sqrt{\lambda+\mu}}}^{\frac{\mu t}{\sqrt{\lambda+\mu}}} e^{-u^{2}} d u \\
& =e^{-\lambda\left(1-\frac{\lambda}{\lambda+\mu}\right) t^{2}} \sum_{n=0}^{\infty} \frac{\lambda^{2 n+1}+\mu^{2 n+1}}{(\lambda+\mu)^{n+1}} \frac{(-1)^{n}}{n !} \frac{1}{2 n+1} t^{2 n+1}
\end{aligned}
$$

Proof. We compute

$$
\begin{aligned}
& \left(e^{-\lambda t^{2}} * e^{-\mu t^{2}}\right)(t)=\int_{0}^{t} e^{-\lambda(t-\tau)^{2}} e^{-\mu \tau^{2}} d \tau= \\
& e^{-\lambda t^{2}} \int_{0}^{t} e^{-(\lambda+\mu)\left(\tau-\frac{\lambda t}{\lambda+\mu}\right)^{2}+\frac{\lambda^{2} t^{2}}{\lambda+\mu}} d \tau=e^{-\lambda\left(1-\frac{\lambda}{\lambda+\mu}\right) t^{2}} \int_{0}^{t} e^{-(\lambda+\mu)\left(\tau-\frac{\lambda t}{\lambda+\mu}\right)^{2}} d \tau
\end{aligned}
$$


with $1-\frac{\lambda}{\lambda+\mu}>0$, and the integral is computed by change of variables as:

$$
\begin{aligned}
& g(t) \equiv \int_{0}^{t} e^{-(\lambda+\mu)\left(\tau-\frac{\lambda t}{\lambda+\mu}\right)^{2}} d \tau \\
& =\frac{1}{\sqrt{\lambda+\mu}} \int_{\frac{-\lambda t}{\sqrt{\lambda+\mu}}}^{\frac{\mu t}{\sqrt{\lambda+\mu}}} e^{-\mathfrak{u}^{2}} d u \quad \text { with } u=\sqrt{\lambda+\mu}\left(\tau-\frac{\lambda t}{\lambda+\mu}\right) .
\end{aligned}
$$

Using Taylor's expansion of $e^{y}$ we compute the integral above as:

$$
\begin{aligned}
& \int_{\frac{-\lambda t}{\sqrt{\lambda+\mu}}}^{\frac{\mu t}{\sqrt{\lambda+\mu}}} e^{-u^{2}} d u=\int_{\frac{-\lambda t}{\sqrt{\lambda+\mu}}}^{\frac{\mu t}{\sqrt{\lambda+\mu}}} \sum_{n=0}^{\infty} \frac{(-1)^{n}}{n !} u^{2 n} d u= \\
& \sum_{n=0}^{\infty} \frac{(-1)^{n}}{n !} \int_{\frac{-\lambda t}{\sqrt{\lambda+\mu}}}^{\frac{\mu t}{\sqrt{\lambda+\mu}}} u^{2 n} d u=\sum_{n=0}^{\infty} \frac{(-1)^{n}}{n !} \frac{\mu^{2 n+1}+\lambda^{2 n+1}}{(\lambda+\mu)^{n+\frac{1}{2}}} \frac{1}{2 n+1} t^{2 n+1} .
\end{aligned}
$$

Corollary 1. The convolutions $e^{-\lambda t^{2}} * e^{-\mu t^{2}}$ for $\lambda, \mu$ positive reals are point-wise limits of elements of $A(\mathbb{R})$.

Proof. Note that any Gaussian functions are point-wise limits of elements of $A(\mathbb{R})$ by Taylor series.

Proposition 2. For $\lambda \in \mathbb{R}$ positive, $n \in \mathbb{N}$, and $t \in \mathbb{R}$,

$$
\text { (1) }\left(t^{n} * e^{-\lambda t^{2}}\right)=\sum_{k=0}^{n}\left(\begin{array}{l}
n \\
k
\end{array}\right) t^{n-k}(-1)^{k} \int_{0}^{t} \tau^{k} e^{-\lambda \tau^{2}} d \tau
$$

with $I_{k} \equiv \int_{0}^{t} \tau^{k} e^{-\lambda \tau^{2}} d \tau$ given by

$$
I_{2 k+1}=-e^{-\lambda t^{2}} \sum_{l=1}^{k} \frac{(2 k) ! !}{(2 \lambda)^{l}(2(k-l+1)) ! !} t^{2(k-l+1)}+\frac{(2 k) ! !}{(2 \lambda)^{k+1}}\left(1-e^{-\lambda t^{2}}\right),
$$

and

$$
\begin{aligned}
I_{2 k}= & -e^{-\lambda t^{2}} \sum_{l=1}^{k} \frac{(2 k-1) ! !}{(2 \lambda)^{l}(2(k-l+1)-1) ! !} t^{2(k-l+1)-1} \\
& +\frac{(2 k-1) ! !}{(2 \lambda)^{k}} \sum_{m=0}^{\infty} \frac{(-\lambda)^{m}}{m !(2 m+1)} t^{2 m+1}
\end{aligned}
$$

Also, for $\mu \in \mathbb{R}$ non-zero and $\lambda \in \mathbb{R}$ positive,

$$
\text { (2) } \quad\left(e^{\mu t} * e^{-\lambda t^{2}}\right)=e^{\frac{\mu^{2}}{4 \lambda}} e^{\mu t} \sum_{n=0}^{\infty} \frac{(-1)^{n} \lambda^{n+\frac{1}{2}}}{n !(2 n+1)}\left\{\left(t+\frac{\mu}{2 \lambda}\right)^{2 n+1}-\left(\frac{\mu}{2 \lambda}\right)^{2 n+1}\right\} \text {. }
$$


Also, for $\mu \in \mathbb{R}$ non-zero and $\lambda \in \mathbb{R}$ positive,

$$
\text { (3) } \quad\left(\sin \mu t * e^{-\lambda t^{2}}\right)=\sin \mu t \int_{0}^{t} \cos \mu \tau e^{-\lambda \tau^{2}} d \tau-\cos \mu t \int_{0}^{t} \sin \mu \tau e^{-\lambda \tau^{2}} d \tau
$$

with

$$
\begin{aligned}
& \int_{0}^{t} \cos \mu \tau e^{-\lambda \tau^{2}} d \tau=\sum_{n=0}^{\infty} \frac{(-\lambda)^{n}}{n !} \int_{0}^{t} \tau^{2 n} \cos \mu \tau d \tau, \\
& \int_{0}^{t} \sin \mu \tau e^{-\lambda \tau^{2}} d \tau=\sum_{n=0}^{\infty} \frac{(-\lambda)^{n}}{n !} \int_{0}^{t} \tau^{2 n} \sin \mu \tau d \tau
\end{aligned}
$$

and each integral term is computed inductively as:

$$
\begin{aligned}
I_{c, 2 n} & \equiv \int_{0}^{t} \tau^{2 n} \cos \mu \tau d \tau \\
& =\frac{1}{\mu} t^{2 n} \sin \mu t+\frac{2 n}{\mu^{2}} t^{2 n-1} \cos \mu \tau-\frac{(2 n)(2 n-1)}{\mu^{2}} I_{c, 2 n-2}, \\
I_{s, 2 n} & \equiv \int_{0}^{t} \tau^{2 n} \sin \mu \tau d \tau \\
& =-\frac{1}{\mu} t^{2 n} \cos \mu t+\frac{2 n}{\mu^{2}} t^{2 n-1} \sin \mu \tau-\frac{(2 n)(2 n-1)}{\mu^{2}} I_{s, 2 n-2},
\end{aligned}
$$

and similarly,

(4) $\left(\cos \mu t * e^{-\lambda t^{2}}\right)=\cos \mu t \int_{0}^{t} \cos \mu \tau e^{-\lambda \tau^{2}} d \tau-\sin \mu t \int_{0}^{t} \sin \mu \tau e^{-\lambda \tau^{2}} d \tau$

with the integrals the same as above.

Proof. For (1) we compute

$$
\left(t^{n} * e^{-\lambda t^{2}}\right)(t)=\int_{0}^{t}(t-\tau)^{n} e^{-\lambda \tau^{2}} d \tau=\sum_{k=0}^{n}\left(\begin{array}{l}
n \\
k
\end{array}\right) t^{n-k}(-1)^{k} \int_{0}^{t} \tau^{k} e^{-\lambda \tau^{2}} d \tau
$$

and the integral is computed by integration by parts as:

$$
\begin{aligned}
I_{k} & \equiv \int_{0}^{t} \tau^{k} e^{-\lambda \tau^{2}} d \tau=\int_{0}^{t} \tau^{k-1}\left\{\frac{1}{-2 \lambda}\left(e^{-\lambda \tau^{2}}\right)^{\prime}\right\} d \tau \\
& =-\frac{1}{2 \lambda} t^{k-1} e^{-\lambda t^{2}}+\frac{k-1}{2 \lambda} I_{k-2}
\end{aligned}
$$


so that, inductively,

$$
\begin{aligned}
I_{2 k+1}= & -\frac{1}{2 \lambda} t^{2 k} e^{-\lambda t^{2}}-\frac{2 k}{(2 \lambda)^{2}} t^{2 k-2} e^{-\lambda t^{2}}-\frac{2 k(2 k-2)}{(2 \lambda)^{3}} t^{2 k-4} e^{-\lambda t^{2}}-\cdots \\
& -\frac{2 k(2 k-2) \cdots 4}{(2 \lambda)^{k}} t^{2} e^{-\lambda t^{2}}+\frac{2 k(2 k-2) \cdots 2}{(2 \lambda)^{k}} I_{1}
\end{aligned}
$$

with

$$
\mathrm{I}_{1} \equiv \int_{0}^{\mathrm{t}} \tau e^{-\lambda \tau^{2}} \mathrm{~d} \tau=\frac{1}{2 \lambda}\left(1-e^{-\lambda \mathrm{t}^{2}}\right)
$$

and

$$
\begin{aligned}
I_{2 k}= & -\frac{1}{2 \lambda} t^{2 k-1} e^{-\lambda t^{2}}-\frac{2 k-1}{(2 \lambda)^{2}} t^{2 k-3} e^{-\lambda t^{2}}-\frac{(2 k-1)(2 k-3)}{(2 \lambda)^{3}} t^{2 k-5} e^{-\lambda t^{2}}-\cdots \\
& -\frac{(2 k-1)(2 k-3) \cdots 3}{(2 \lambda)^{k}} t e^{-\lambda t^{2}}+\frac{(2 k-1)(2 k-3) \cdots 1}{(2 \lambda)^{k}} I_{O}
\end{aligned}
$$

with

$$
\begin{aligned}
I_{0} & \equiv \int_{0}^{t} e^{-\lambda \tau^{2}} d \tau=\int_{0}^{t} \sum_{m=0}^{\infty} \frac{(-\lambda)^{m}}{m !} \tau^{2 m} d \tau \\
& =\sum_{m=0}^{\infty} \frac{(-\lambda)^{m}}{m !} \int_{0}^{t} \tau^{2 m} d \tau=\sum_{m=0}^{\infty} \frac{(-\lambda)^{m}}{m !(2 m+1)} t^{2 m+1}
\end{aligned}
$$

For (2) we compute

$$
e^{\mu t} * e^{-\lambda t^{2}}=\int_{0}^{t} e^{\mu(t-\tau)} e^{-\lambda \tau^{2}} d \tau=e^{\frac{\mu^{2}}{4 \lambda}} e^{\mu t} \int_{0}^{t} e^{-\lambda\left(\tau+\frac{\mu}{2 \lambda}\right)^{2}} d \tau
$$

By change of variables as $u=\sqrt{\lambda}\left(\tau+\frac{\mu}{2 \lambda}\right)$, the integral above is computed as

$$
\begin{aligned}
& \int_{0}^{t} e^{-\lambda\left(\tau+\frac{\mu}{2 \lambda}\right)^{2}} d \tau=\int_{\sqrt{\lambda}\left(\frac{\mu}{2 \lambda}\right)}^{\sqrt{\lambda}\left(t+\frac{\mu}{2 \lambda}\right)} e^{-u^{2}} d u=\int_{\sqrt{\lambda}\left(\frac{\mu}{2 \lambda}\right)}^{\sqrt{\lambda}\left(t+\frac{\mu}{2 \lambda}\right)} \sum_{n=0}^{\infty} \frac{1}{n !}\left(-u^{2}\right)^{n} d u \\
& =\sum_{n=0}^{\infty} \frac{(-1)^{n}}{n !} \int_{\sqrt{\lambda}\left(\frac{\mu}{2 \lambda}\right)}^{\sqrt{\lambda}\left(t+\frac{\mu}{2 \lambda}\right)} u^{2 n} d u=\sum_{n=0}^{\infty} \frac{(-1)^{n} \lambda^{n+\frac{1}{2}}}{n !(2 n+1)}\left\{\left(t+\frac{\mu}{2 \lambda}\right)^{2 n+1}-\left(\frac{\mu}{2 \lambda}\right)^{2 n+1}\right\} .
\end{aligned}
$$

For (3) we next compute

$$
\begin{aligned}
& \left(\sin \mu t * e^{-\lambda t^{2}}\right)(t)=\int_{0}^{t} \sin \mu(t-\tau) e^{-\lambda t^{2}} d \tau \\
& =\sin \mu t \int_{0}^{t} \cos \mu \tau e^{-\lambda \tau^{2}} d \tau-\cos \mu t \int_{0}^{t} \sin \mu \tau e^{-\lambda \tau^{2}} d \tau,
\end{aligned}
$$


and the first integral is computed as:

$$
\int_{0}^{t} \cos \mu \tau e^{-\lambda \tau^{2}} d \tau=\int_{0}^{t} \cos \mu \tau \sum_{n=0}^{\infty} \frac{1}{n !}\left(-\lambda \tau^{2}\right)^{n} d \tau=\sum_{n=0}^{\infty} \frac{(-\lambda)^{n}}{n !} \int_{0}^{t} \tau^{2 n} \cos \mu \tau d \tau
$$

and each integral term is computed inductively as:

$$
\begin{aligned}
I_{c, 2 n} & \equiv \int_{0}^{t} \tau^{2 n} \cos \mu \tau d \tau \\
& =\frac{1}{\mu} t^{2 n} \sin \mu t+\frac{2 n}{\mu^{2}} t^{2 n-1} \cos \mu \tau-\frac{(2 n)(2 n-1)}{\mu^{2}} I_{c, 2 n-2}
\end{aligned}
$$

by using integration by parts. Similarly, we obtain

$$
\int_{0}^{t} \sin \mu \tau e^{-\lambda \tau^{2}} d \tau=\sum_{n=0}^{\infty} \frac{(-\lambda)^{n}}{n !} \int_{0}^{t} \tau^{2 n} \sin \mu \tau d \tau
$$

and

$$
\begin{aligned}
I_{s, 2 n} & \equiv \int_{0}^{t} \tau^{2 n} \sin \mu \tau d \tau \\
& =-\frac{1}{\mu} t^{2 n} \cos \mu t+\frac{2 n}{\mu^{2}} t^{2 n-1} \sin \mu \tau-\frac{(2 n)(2 n-1)}{\mu^{2}} I_{s, 2 n-2} .
\end{aligned}
$$

Remark. We omit the case of convolutions with general monomials such as multiples $t^{\mathrm{n}} e^{\mu \mathrm{t}} \sin \mu \mathrm{t}$, but which are certainly computable by the similar computation technique as above, with some more integration. Refer to [3].

\section{Algebraic structure with Gaussian functions}

We denote by $A^{w}(\mathbb{R})$ the set of all functions on $\mathbb{R}$ that are written as point-wise limits in $\mathbb{R}$ of elements of $A(\mathbb{R})$.

Lemma 3.1. $A^{w}(\mathbb{R})$ is an algebra over $\mathbb{R}$ with point-wise multiplication. Also, $A^{w}(\mathbb{R})$ is an algebra over $\mathbb{R}$ with convolution.

Proof. Suppose that $f, g \in A^{w}(\mathbb{R})$ with $f=\lim f_{n}, g=\lim g_{n}(n \rightarrow \infty)$ point-wise limits of $f_{n}, g_{n} \in A^{w}(\mathbb{R})$. Since the limits at any $t \in \mathbb{R}$ are finite, then

$$
(f \cdot g)(t)=\lim f_{n}(t) \cdot \lim g_{n}(t)=\lim \left(f_{n} \cdot g_{n}\right)(t) \in \mathbb{R},
$$

and hence, $f \cdot g \in A^{w}(\mathbb{R})$. 
Note also that we define the convolution of $f, g \in A^{w}(\mathbb{R})$ as

$$
f * g=\lim \left(f_{\mathfrak{n}} * g_{\mathfrak{n}}\right) \in A^{w}(\mathbb{R}),
$$

where it is shown in general $([3])$ that $A(\mathbb{R})$ is closed under convolution. This is well defined, because if $f=\lim h_{n}$ and $g=\lim k_{n}$ another limits with $h_{n}, k_{n} \in A(\mathbb{R})$, and since the limits at any $t \in \mathbb{R}$ are finite, then

$$
\begin{aligned}
\left(f_{n} * g_{n}\right)(t)-\left(h_{n} * k_{n}\right)(t)= & \left(\left(f_{n}-f\right) * g_{n}\right)(t)+\left(f *\left(g_{n}-g\right)\right)(t) \\
& +\left(\left(f-h_{n}\right) * g\right)(t)+\left(h_{n} *\left(g-k_{n}\right)\right)(t),
\end{aligned}
$$

and the right hand side goes to zero as $\mathrm{n} \rightarrow \infty$ by applying the dominated convergence theorem to each convolution, and hence,

$$
\begin{aligned}
& \left|\lim \left(f_{n} * g_{n}\right)-\lim \left(h_{n} * k_{n}\right)\right| \\
& \leq\left|\lim \left(f_{n} * g_{n}\right)-f_{n} * g_{n}\right|+\left|f_{n} * g_{n}-h_{n} * k_{n}\right|+\left|h_{n} * k_{n}-\lim \left(h_{n} * k_{n}\right)\right|,
\end{aligned}
$$

which go to zero as $n \rightarrow \infty$.

We denote by $G(\mathbb{R})$ the algebra generated by $A(\mathbb{R})$ and the set $\left\{e^{-\lambda t^{2}} \mid \lambda \in \mathbb{R}, \lambda>0\right\}$, with either point-wise multiplication or convolution as a product.

Corollary 2. The algebra $G(\mathbb{R})$ is contained in $A^{w}(\mathbb{R})$ and is a subalgebra of $A^{w}(\mathbb{R})$ with either point-wise multiplication or convolution.

We denote by $R_{0}(\mathbb{C})$ the algebra of rational functions on $\mathbb{C}$ vanishing at infinity with point-wise multiplication and by $E(\mathbb{C})$ the algebra generated by both $R_{0}(\mathbb{C})$ and the set $\left\{e^{\lambda s^{2}} \mid \lambda \in \mathbb{R}, \lambda>0\right\}$ of inverse Gaussian functions on $\mathbb{C}$ with s complex variable.

Corollary 3. The algebra $G(\mathbb{R})$ with convolution is isomorphic to the algebra $E(\mathbb{C})$ by the Laplace transform and by the inverse Laplace transform.

Proof. Note that $\mathrm{L}(\mathrm{f} * \mathrm{~g})=\mathrm{L}(\mathrm{f}) \cdot \mathrm{L}(\mathrm{g})$ and $\mathrm{L}$ is injective for continuous functions on $\mathbb{R}$.

\section{Adjoining the delta function}

We consider the Dirac delta function. Define the delta function as

$$
\delta(t)=\lim _{\lambda \rightarrow \infty} g_{\lambda}(t) \equiv \lim _{\lambda \rightarrow \infty} 2 \sqrt{\frac{\lambda}{\pi}} e^{-\lambda t^{2}}= \begin{cases}\infty & t=0 \\ 0 & t \neq 0\end{cases}
$$

with $\lambda \in \mathbb{R}$ positive. Note that the delta function has the meaning in integration only in the weak sense, i.e. as the weak limit of $g_{\lambda}(t)$ by taking the inner product or the convolution with test 
functions, and the delta function is also viewed as a distribution, i.e., a functional (or a scalarvalued linear map) on the linear space of test functions. Also, one can take other functions of different types to define the delta function.

Note that $\int_{-\infty}^{\infty} g_{\lambda}(t) d t=2$ but we need to have the next:

Lemma 4.1. The functions $g_{\lambda}$ are in $G(\mathbb{R})$, and for $s \in \mathbb{C}$,

$$
\mathrm{L}\left(\mathrm{g}_{\lambda}\right)(\mathrm{s})=\mathrm{e}^{\frac{\mathrm{s}^{2}}{4 \lambda}},
$$

which goes to 1 as $\lambda \rightarrow \infty$.

Proof. Use Lemma 2.1.

Lemma 4.2. For $s \in \mathbb{C}$, we have

$$
\mathrm{L}\left(\mathrm{g}_{\lambda} * \mathrm{~g}_{\mu}\right)(\mathrm{s})=\mathrm{e}^{\frac{(\lambda+\mu) s^{2}}{4 \lambda \mu}},
$$

which goes to 1 as $\lambda \rightarrow \infty$ and $\mu \rightarrow \infty$.

Proof. Use Lemma 4.1 and $\mathrm{L}\left(\mathrm{g}_{\lambda} * \mathrm{~g}_{\mu}\right)=\mathrm{L}\left(\mathrm{g}_{\lambda}\right) \cdot \mathrm{L}\left(\mathrm{g}_{\mu}\right)$.

Proposition 3. For positive $\mu, \lambda \in \mathbb{R}$,

$$
\text { (1) } \lim _{\lambda \rightarrow \infty}\left(e^{-\mu t^{2}} * g_{\lambda}\right)(t)= \begin{cases}e^{-\mu t^{2}} & \text { if } t>0, \\ 0 & \text { if } t=0, \\ -e^{-\mu t^{2}} & \text { if } t<0\end{cases}
$$

and for $n \in \mathbb{N}$ and $\lambda>0$,

$$
\text { (2) } \lim _{\lambda \rightarrow \infty}\left(t^{n} * g_{\lambda}\right)(t)= \begin{cases}t^{n} & \text { if } t>0, \\ 0 & \text { if } t=0 \\ -t^{n} & \text { if } t<0\end{cases}
$$

and for $\mu \in \mathbb{R}$ and $\lambda>0$,

$$
\text { (3) } \lim _{\lambda \rightarrow \infty}\left(e^{\mu t} * g_{\lambda}\right)(t)= \begin{cases}e^{\mu t} & \text { if } t>0 \\ 0 & \text { if } t=0 \\ -e^{\mu t} & \text { if } t<0\end{cases}
$$

and for $\mu \in \mathbb{R}$ and $\lambda>0$,

$$
\text { (4) } \lim _{\lambda \rightarrow \infty}\left(\sin \mu t * g_{\lambda}\right)(t)= \begin{cases}\sin \mu t & \text { if } t>0, \\ 0 & \text { if } t=0, \\ -\sin \mu t & \text { if } t<0\end{cases}
$$


and

$$
\text { (5) } \lim _{\lambda \rightarrow \infty}\left(\cos \mu t * g_{\lambda}\right)(t)= \begin{cases}\cos \mu t & \text { if } t>0 \\ 0 & \text { if } t=0 \\ -\cos \mu t & \text { if } t<0\end{cases}
$$

Proof. The first equation (1) follows from the first equation in Proposition 1 by taking the limit with respect to $\lambda$.

For (2) we next use Proposition 2. It follows that

$$
\lim _{\lambda \rightarrow \infty}\left(t^{n} * g_{\lambda}\right)(t)=\lim _{\lambda \rightarrow \infty} t^{n} \int_{0}^{t} g_{\lambda}(\tau) d \tau,
$$

namely, other terms in the convolution go to zero as $\lambda \rightarrow \infty$, and then

$$
\int_{0}^{t} g_{\lambda}(\tau) d \tau=\int_{0}^{\sqrt{\lambda} t} \frac{2}{\sqrt{\pi}} e^{-u^{2}} d u \quad(u=\sqrt{\lambda} \tau)
$$

which goes to

$$
\begin{cases}1 & \text { if } t>0, \\ 0 & \text { if } t=0, \\ -1 & \text { if } t<0\end{cases}
$$

For (3), it also follows as in parts of the proof of Proposition 2 that

$$
\begin{aligned}
\left(e^{\mu t} * g_{\lambda}\right)(t) & =e^{\frac{\mu^{2}}{4 \lambda}} e^{\mu t} \int_{0}^{t} 2 \sqrt{\frac{\lambda}{\pi}} e^{-\lambda\left(\tau+\frac{\mu}{2 \lambda}\right)^{2}} d \tau \\
& =e^{\frac{\mu^{2}}{4 \lambda}} e^{\mu t} \int_{\frac{\mu}{2 \sqrt{\lambda}}}^{\sqrt{\lambda}\left(t+\frac{\mu}{2 \lambda}\right)} \frac{2}{\sqrt{\pi}} e^{-u^{2}} d u \quad\left(u=\sqrt{\lambda}\left(\tau+\frac{\mu}{2 \lambda}\right)\right),
\end{aligned}
$$

which goes to

$$
\begin{cases}e^{\mu t} & \text { if } t>0 \\ 0 & \text { if } t=0, \\ -e^{\mu t} & \text { if } t<0\end{cases}
$$

For (4) we next compute by allowing complex coefficients for this case and using the Euler formula as:

$$
\begin{aligned}
\left(\sin \mu t * g_{\lambda}\right)(t) & =\left(\frac{e^{\mu t i}-e^{-\mu t i}}{2 i} * g_{\lambda}\right)(t) \\
& =\frac{1}{2 i} \int_{0}^{t} e^{\mu(t-\tau) i} g_{\lambda}(\tau) d \tau-\frac{1}{2 i} \int_{0}^{t} e^{-\mu(t-\tau) i} g_{\lambda}(\tau) d \tau \\
& =\frac{e^{\mu t i}}{2 i} \int_{0}^{t} e^{-\mu \tau i} g_{\lambda}(\tau) d \tau-\frac{e^{-\mu t i}}{2 i} \int_{0}^{t} e^{\mu \tau i} g_{\lambda}(\tau) d \tau
\end{aligned}
$$


and the first integral is converted to

$$
\begin{aligned}
& \int_{0}^{t} 2 \sqrt{\frac{\lambda}{\pi}} e^{-\lambda\left(\tau+\frac{\mu i}{2 \lambda}\right)^{2}-\frac{\mu^{2}}{4 \lambda}} d \tau \\
& =e^{-\frac{\mu^{2}}{4 \lambda}} \int_{\frac{i \mu}{2 \sqrt{\lambda}}}^{\sqrt{\lambda}\left(t+\frac{\mu i}{2 \lambda}\right)} \frac{2}{\sqrt{\pi}} e^{-u^{2}} d u \quad\left(u=\sqrt{\lambda}\left(\tau+\frac{\mu i}{2 \lambda}\right)\right)
\end{aligned}
$$

which goes to

$$
\begin{cases}1 & \text { if } t>0 \\ 0 & \text { if } t=0 \\ -1 & \text { if } t<0\end{cases}
$$

as $\lambda \rightarrow \infty$ via Cauchy integral theorem. Similarly, the second integral above is converted to

$$
\begin{aligned}
& \int_{0}^{t} 2 \sqrt{\frac{\lambda}{\pi}} e^{-\lambda\left(\tau-\frac{\mu i}{2 \lambda}\right)^{2}-\frac{\mu^{2}}{4 \lambda}} d \tau \\
& =e^{-\frac{\mu^{2}}{4 \lambda}} \int_{\frac{i \mu}{2 \sqrt{\lambda}}}^{\sqrt{\lambda}\left(t-\frac{\mu i}{2 \lambda}\right)} \frac{2}{\sqrt{\pi}} e^{-u^{2}} d u \quad\left(u=\sqrt{\lambda}\left(\tau-\frac{\mu i}{2 \lambda}\right)\right)
\end{aligned}
$$

which goes to

$$
\begin{cases}1 & \text { if } t>0 \\ 0 & \text { if } t=0 \\ -1 & \text { if } t<0\end{cases}
$$

as $\lambda \rightarrow \infty$. Therefore, we obtain one of the last two equations in the statement. The other case (5) is obtained similarly, by $\cos \mu t=\frac{e^{\mu t i}+e^{-\mu t i}}{2}$.

\section{$5 \quad$ Algebraic structure with the delta function}

We denote by $G^{\delta}(\mathbb{R})$ the algebra generated by $G(\mathbb{R})$ and the delta function $\delta(t)$ with convolution, where we assume that $f * \delta=f$ on $[0, \infty)$ for any $f \in G(\mathbb{R})$ and $\delta * \delta=\delta$ on $[0, \infty)$, which follows from our definition of the delta function in the weak sense, so that $G^{\delta}(\mathbb{R})$ restricted to $[0, \infty)$ is isomorphic to the unitization of $G(\mathbb{R})$ by $\mathbb{R}$, restricted to $[0, \infty)$, as a real algebra. We define the Laplace transform for $\delta$ as

$$
\mathrm{L}(\delta(\mathrm{t}))(\mathrm{s})=1(\mathrm{~s})
$$

the unit function on $\mathbb{C}$.

Note that $G^{\delta}(\mathbb{R})$ is also viewed as the generated by $G(\mathbb{R})$ and the point-wise limit function of Gaussian functions on $\mathbb{R}$.

We denote by $E^{1}(\mathbb{C})$ the algebra generated by $E(\mathbb{C})$ and the unit function 1 on $\mathbb{C}$ with pointwise multiplication. 
Corollary 4. The algebra $G^{\delta}(\mathbb{R})$ with convolution, restricted to $[0, \infty)$, is isomorphic to the algebra $E^{1}(\mathbb{C})$ by the Laplace transform and by the inverse Laplace transform.

Remark. Our Proposition 3 says that the convolution $f * \delta$ on $\mathbb{R}$ for $f \in G^{\delta}(\mathbb{R})$ is defined as

$$
(f * \delta)(t)= \begin{cases}f(t) & \text { if } t>0 \\ 0 & \text { if } t=0 \\ -f(t) & \text { if } t<0\end{cases}
$$

\section{Adjoining the shifted delta functions}

It is also defined as that for $t \in \mathbb{R}$ and $s \in \mathbb{C}$,

$$
\mathrm{L}(\delta(\mathrm{t}-\mu))=e^{-\mu s}
$$

for $\mu>0$. We extend this definition for $\mu \in \mathbb{R}$. Indeed, check that

Lemma 6.1. We have

$$
\lim _{\lambda \rightarrow \infty} L\left(g_{\lambda}(t-\mu)\right)(s)=e^{-\mu s}
$$

for any $\mu \in \mathbb{R}$.

Proof. We compute

$$
\begin{aligned}
L\left(g_{\lambda}(t-\mu)\right)(s) & =\int_{0}^{\infty} e^{-s t} 2 \sqrt{\frac{\lambda}{\pi}} e^{-\lambda(t-\mu)^{2}} d t \\
& =e^{-\mu s} e^{\frac{s^{2}}{4 \lambda}} \int_{0}^{\infty} e^{-\lambda\left(t+\frac{s}{2 \lambda}-\mu\right)^{2}} 2 \sqrt{\frac{\lambda}{\pi}} d t
\end{aligned}
$$

and changing variables as $u=\sqrt{\lambda}\left(t+\frac{s}{2 \lambda}-u\right)$ and using Cauchy integral theorem and taking the limit as $\lambda \rightarrow \infty$ we obtain the result in the statement.

Lemma 6.2. We have

$$
\lim _{\lambda \rightarrow \infty} L\left(g_{\lambda}(t-\mu) * g_{\lambda}(t-\rho)\right)(s)=e^{-(\mu+\rho) s}
$$

for any $\mu, \rho \in \mathbb{R}$. It follows by defining the left hand side to be equal to

$$
\mathrm{L}(\delta(\mathrm{t}-\mu) * \delta(\mathrm{t}-\rho))(\mathrm{s})=\mathrm{e}^{-(\mu+\rho) s} .
$$

Proof. Note that

$$
\mathrm{L}\left(\mathrm{g}_{\lambda}(\mathrm{t}-\mu) * \mathrm{~g}_{\lambda}(\mathrm{t}-\rho)\right)(\mathrm{s})=\mathrm{L}\left(\mathrm{g}_{\lambda}(\mathrm{t}-\mu)\right)(\mathrm{s}) \cdot \mathrm{L}\left(\mathrm{g}_{\lambda}(\mathrm{t}-\rho)\right)(\mathrm{s})
$$

and the both factors are computed in the proof of Lemma 6.1. 
Proposition 4. For positive $\mu, \lambda \in \mathbb{R}$ and $\rho \in \mathbb{R}$, if $\rho>0$, then

$$
(1)_{+} \lim _{\lambda \rightarrow \infty}\left(e^{-\mu t^{2}} * g_{\lambda}(t-\rho)\right)(t)= \begin{cases}2 e^{-\mu(t-\rho)^{2}} & t>\rho \\ 1 & t=\rho \\ 0 & t<\rho\end{cases}
$$

and if $\rho<0$, then

$$
\text { (1) } \lim _{\lambda \rightarrow \infty}\left(e^{-\mu t^{2}} * g_{\lambda}(t-\rho)\right)(t)= \begin{cases}0 & t>\rho, \\ -1 & t=\rho, \\ -2 e^{-\mu(t-\rho)^{2}} & t<\rho\end{cases}
$$

and for $n \in \mathbb{N}$ and $\rho \in \mathbb{R}$, if $\rho>0$, then

$$
(2)_{+} \lim _{\lambda \rightarrow \infty}\left(t^{n} * g_{\lambda}(t-\rho)\right)(t)= \begin{cases}2 t^{n} & t>\rho \\ \rho^{n} & t=\rho \\ 0 & t<\rho\end{cases}
$$

and if $\rho<0$, then

$$
\text { (2) }-\lim _{\lambda \rightarrow \infty}\left(t^{n} * g_{\lambda}(t-\rho)\right)(t)= \begin{cases}0 & t>\rho, \\ -\rho^{n} & t=\rho, \\ -2 t^{n} & t<\rho ;\end{cases}
$$

and for $\mu \in \mathbb{R}$, if $\rho>0$, then

$$
(3)_{+} \lim _{\lambda \rightarrow \infty}\left(e^{\mu t} * g_{\lambda}(t-\rho)\right)(t)= \begin{cases}2 e^{\mu(t-\rho)} & \text { if } t>\rho, \\ 1 & \text { if } t=\rho, \\ 0 & \text { if } t<\rho,\end{cases}
$$

and if $\rho<0$, then

$$
\text { (3) } \lim _{\lambda \rightarrow \infty}\left(e^{\mu t} * g_{\lambda}(t-\rho)\right)(t)= \begin{cases}0 & \text { if } t>\rho, \\ -1 & \text { if } t=\rho, \\ -2 e^{\mu(t-\rho)} & \text { if } t<\rho ;\end{cases}
$$

and for $\mu \in \mathbb{R}$, if $\rho>0$, then

$$
\text { (4) } \lim _{\lambda \rightarrow \infty}\left(\sin \mu t * g_{\lambda}(t-\rho)(t)= \begin{cases}2 \sin \mu(t-\rho) & \text { if } t>\rho, \\ 0 & \text { if } t=\rho, \\ 0 & \text { if } t<\rho,\end{cases}\right.
$$

and if $\rho<0$, then

$$
\text { (4)_- } \lim _{\lambda \rightarrow \infty}\left(\sin \mu t * g_{\lambda}(t-\rho)(t)= \begin{cases}0 & \text { if } t>\rho, \\ 0 & \text { if } t=\rho, \\ -2 \sin \mu(t-\rho) & \text { if } t<\rho ;\end{cases}\right.
$$


and if $\rho>0$, then

$$
(5)_{+} \lim _{\lambda \rightarrow \infty}\left(\cos \mu t * g_{\lambda}(t-\rho)\right)(t)= \begin{cases}2 \cos \mu(t-\rho) & \text { if } t>\rho, \\ 1 & \text { if } t=\rho, \\ 0 & \text { if } t<\rho,\end{cases}
$$

and if $\rho>0$, then

$$
\text { (5) } \lim _{\lambda \rightarrow \infty}\left(\cos \mu t * g_{\lambda}(t-\rho)\right)(t)= \begin{cases}0 & \text { if } t>\rho, \\ -1 & \text { if } t=\rho, \\ -2 \cos \mu(t-\rho) & \text { if } t<\rho .\end{cases}
$$

Proof. For $(1)_{ \pm}$we compute

$$
\begin{aligned}
\left(e^{-\mu t^{2}} * g_{\lambda}(t-\rho)\right)(t) & =\int_{0}^{t} 2 \sqrt{\frac{\lambda}{\pi}} e^{-\lambda(t-\tau-\rho)^{2}} e^{-\mu \tau^{2}} d \tau \\
& =e^{\frac{-\lambda \mu}{\lambda+\mu}(t-\rho)^{2}} \int_{0}^{t} 2 \sqrt{\frac{\lambda}{\pi}} e^{-(\lambda+\mu)\left(\tau-\frac{\lambda(t-\rho)}{\lambda+\mu}\right)^{2}} d \tau \\
& =e^{\frac{-\lambda \mu}{\lambda+\mu}(t-\rho)^{2}} \int_{-\frac{\lambda(t-\rho)}{\sqrt{\lambda+\mu}}}^{\sqrt{\lambda+\mu}\left(t-\frac{\lambda(t-\rho)}{\lambda+\mu}\right)} 2 \sqrt{\frac{\lambda}{\pi(\lambda+\mu)}} e^{-u^{2}} d u
\end{aligned}
$$

where $\mathfrak{u}=\sqrt{\lambda+\mu}\left(\tau-\frac{\lambda(t-\rho)}{\lambda+\mu}\right)$ and if $\tau=t$, then $\mathfrak{u}=\frac{\mu t+\lambda \rho}{\sqrt{\lambda+\mu}}$, which goes to $\infty$ as $\lambda \rightarrow \infty$ for $\rho>0$. If $t>\rho$, then $-\frac{\lambda(t-\rho)}{\sqrt{\lambda+\mu}}$ goes to $-\infty$ and if $t<\rho$, then it goes to $\infty$. Therefore, if $\rho>0$, then

$$
\lim _{\lambda \rightarrow \infty}\left(e^{-\mu t^{2}} * g_{\lambda}(t-\rho)\right)(t)= \begin{cases}2 e^{-\mu(t-\rho)^{2}} & t>\rho \\ 1 & t=\rho \\ 0 & t<\rho\end{cases}
$$

and if $\rho<0$, then

$$
\lim _{\lambda \rightarrow \infty}\left(e^{-\mu t^{2}} * g_{\lambda}(t-\rho)\right)(t)= \begin{cases}0 & t>\rho, \\ -1 & t=\rho, \\ -2 e^{-\mu(t-\rho)^{2}} & t<\rho,\end{cases}
$$

For $(2)_{ \pm}$we next compute

$$
\left(t^{n} * g_{\lambda}(t-\rho)\right)(t)=\sum_{k=0}^{n}\left(\begin{array}{l}
n \\
k
\end{array}\right) t^{n-k}(-1)^{k} \int_{0}^{t} \tau^{k} 2 \sqrt{\frac{\lambda}{\pi}} e^{-\lambda(\tau-\rho)^{2}} d \rho
$$

and the following integrals are converted as:

$$
\int_{0}^{t} \tau^{k} e^{-\lambda(\tau-\rho)^{2}} d \tau=\int_{-\sqrt{\lambda} \rho}^{\sqrt{\lambda}(t-\rho)}\left(\rho+\frac{u}{\sqrt{\lambda}}\right)^{k} e^{-u^{2}} \frac{d u}{\sqrt{\lambda}}
$$


where $u=\sqrt{\lambda}(\tau-\rho)$. We then use Proposition 2 and it follows that if $\rho>0$, then

$$
\lim _{\lambda \rightarrow \infty}\left(t^{n} * g_{\lambda}(t-\rho)\right)(t)= \begin{cases}2 t^{n} & t>\rho, \\ \rho^{n} & t=\rho, \\ 0 & t<\rho,\end{cases}
$$

and if $\rho<0$, then

$$
\lim _{\lambda \rightarrow \infty}\left(t^{n} * g_{\lambda}(t-\rho)\right)(t)= \begin{cases}0 & t>\rho, \\ -\rho^{n} & t=\rho, \\ -2 t^{n} & t<\rho .\end{cases}
$$

For $(3)_{ \pm}$we next compute

$$
\begin{aligned}
\left(e^{\mu t} * g_{\lambda}(t-\rho)\right)(t) & =\int_{0}^{t} e^{\mu(t-\tau)} 2 \sqrt{\frac{\lambda}{\pi}} e^{-\lambda(\tau-\rho)^{2}} d \tau \\
& =e^{\frac{\mu^{2}-4 \lambda \rho \mu}{4 \lambda}} e^{\mu t} \int_{0}^{t} 2 \sqrt{\frac{\lambda}{\pi}} e^{-\lambda\left(\tau+\frac{\mu-2 \lambda \rho}{2 \lambda}\right)^{2}} d \tau \\
& =e^{\frac{\mu^{2}-4 \lambda \rho \mu}{4 \lambda}} e^{\mu t} \int_{\frac{\mu-2 \lambda \rho}{2 \sqrt{\lambda}}}^{\sqrt{\lambda}\left(t-\rho+\frac{\mu}{2 \lambda}\right)} \frac{2}{\sqrt{\pi}} e^{-u^{2}} d u \quad\left(u=\sqrt{\lambda}\left(\tau+\frac{\mu-2 \lambda \rho}{2 \lambda}\right)\right),
\end{aligned}
$$

which goes to, if $\rho>0$, then

$$
\begin{cases}2 e^{\mu(t-\rho)} & \text { if } t>\rho \\ 1 & \text { if } t=\rho \\ 0 & \text { if } t<\rho\end{cases}
$$

as $\lambda \rightarrow \infty$, and if $\rho<0$, then

$$
\begin{cases}0 & \text { if } t>\rho \\ -1 & \text { if } t=\rho \\ -2 e^{\mu(t-\rho)} & \text { if } t<\rho .\end{cases}
$$

For $(4)_{ \pm}$we finally compute by allowing complex coefficients for this case and using the Euler formula as:

$$
\begin{aligned}
\left(\sin \mu t * g_{\lambda}(t-\rho)\right)(t) & =\left(\frac{e^{\mu t i}-e^{-\mu t i}}{2 i} * g_{\lambda}(t-\rho)\right)(t) \\
& =\frac{1}{2 i} \int_{0}^{t} e^{\mu(t-\tau) i} g_{\lambda}(\tau-\rho) d \tau-\frac{1}{2 i} \int_{0}^{t} e^{-\mu(t-\tau) i} g_{\lambda}(\tau-\rho) d \tau \\
& =\frac{e^{\mu t i}}{2 i} \int_{0}^{t} e^{-\mu \tau i} g_{\lambda}(\tau-\rho) d \tau-\frac{e^{-\mu t i}}{2 i} \int_{0}^{t} e^{\mu \tau i} g_{\lambda}(\tau-\rho) d \tau
\end{aligned}
$$


and the first integral is converted to

$$
\begin{aligned}
& e^{-\rho \mu i-\frac{\mu^{2}}{4 \lambda}} \int_{0}^{t} 2 \sqrt{\frac{\lambda}{\pi}} e^{-\lambda\left(\tau-\rho+\frac{\mu i}{2 \lambda}\right)^{2}} d \tau \\
& =e^{-\rho \mu i-\frac{\mu^{2}}{4 \lambda}} \int_{\sqrt{\lambda}\left(-\rho+\frac{\mu i}{2 \lambda}\right)}^{\sqrt{\lambda}\left(t-\rho+\frac{\mu i}{2 \lambda}\right)} \frac{2}{\sqrt{\pi}} e^{-u^{2}} d u \quad\left(u=\sqrt{\lambda}\left(\tau-\rho+\frac{\mu i}{2 \lambda}\right)\right)
\end{aligned}
$$

which goes to, if $\rho>0$, then,

$$
\begin{cases}2 e^{-\rho \mu i} & \text { if } t>\rho \\ e^{-\rho \mu i} & \text { if } t=\rho, \\ 0 & \text { if } t<\rho\end{cases}
$$

as $\lambda \rightarrow \infty$ via Cauchy integral theorem, and if $\rho<0$, then,

$$
\begin{cases}0 & \text { if } t>\rho \\ -e^{-\rho \mu i} & \text { if } t=\rho \\ -2 e^{-\rho \mu i} & \text { if } t<\rho .\end{cases}
$$

Similarly, the second integral above is converted to have the similar limits as above, where $e^{-\rho \mu i}$ is replaced with $e^{\rho \mu i}$ since $u$ is replaced with $u=\sqrt{\lambda}\left(\tau-\rho-\frac{\mu i}{2 \lambda}\right)$. Therefore, we obtain one of the last two equations in the statement. The other case $(5)_{ \pm}$is obtained similarly, by $\cos \mu t=$ $\frac{e^{\mu t i}+e^{-\mu t i}}{2}$.

Remark. The Heaviside unit function $u(t)$ on $\mathbb{R}$ is defined by $u(t)=1$ if $t>0$ and $u(t)=0$ if $t<0$ as well as $u(0)=\frac{1}{2}$. For $\rho \in \mathbb{R}$ positive, the shifted Heaviside unit function $u(t-\rho)$ is defined similarly. It is known that

$$
\mathrm{L}(\mathrm{u}(\mathrm{t}-\rho))(\mathrm{s})=\frac{\mathrm{e}^{-\rho s}}{\mathrm{~s}} .
$$

We may extend the definition of $u(t-\rho)$ for $\rho<0$. But we always have

$$
\mathrm{L}(\mathrm{u}(\mathrm{t}-\rho))(\mathrm{s})=\frac{1}{\mathrm{~s}}, \quad \rho \leq 0 .
$$

Note also that

$$
L^{-1}\left(\frac{1}{s} \cdot e^{-\rho s}\right)=\int_{0}^{t} 1 \cdot \delta(\tau-\rho) d \tau= \begin{cases}1 & t>\rho \\ 0 & t<\rho,\end{cases}
$$

where note that this shifted delta function comes from the usual Gaussian functions, not equal to ours multiplied by 2 . 


\section{Algebraic structure with the shifted delta functions}

We denote by $G^{s \delta}(\mathbb{R})$ the algebra generated by $G(\mathbb{R})$ and the shifted delta functions $\delta(t-\mu)$ for $\mu \in \mathbb{R}$, with convolution.

For convenience, we define $G^{s \delta}\left(\mathbb{R}_{+}\right)$to be the algebra generated by $G^{\delta}(\mathbb{R})$ and the positive shifted delta functions $\delta(t-\mu)$ for $\mu>0$, restricted to $[0, \infty)$. We define the following convolution as

$$
(f * \delta(t-\mu))(t)=u(t-\mu) f(t-\mu)
$$

for $f \in G^{s \delta}\left(\mathbb{R}_{+}\right)$and $\mu>0$, where $u(t-\mu)$ is the shifted Heaviside unit function on $[0, \infty)$. Note that this definition is not perfectly compatible with Proposition 4 but either changing $g_{\lambda}(t-\mu)$ with it multiplied by $\frac{1}{2}$ on $[0, \infty)$ or cutting off functions to be zero on $(-\infty, 0)$ make sense almost everywhere. In fact, such a scalar multiplication is allowed to define the convolution with the shifted delta functions in the weak sense.

We denote by $E^{s 1}(\mathbb{C})$ the algebra generated by $E^{1}(\mathbb{C})$ and the set $\left\{e^{-\rho s} \mid \rho>0\right\}$, with pointwise mulitiplication.

We define the Laplace transform $L(f * g)$ to be $L(f) \cdot L(g)$ for $f, g \in G^{s \delta}\left(\mathbb{R}_{+}\right)$.

Note that it is known as the first shift law that

$$
\mathrm{L}(\boldsymbol{u}(\mathrm{t}-\mu) \mathrm{f}(\mathrm{t}-\mu))=e^{-\mu s} \mathrm{~L}(\mathrm{f})(\mathrm{s})
$$

and $\mathrm{L}(\delta(\mathrm{t}-\mu))=e^{-\mu s}$.

Corollary 5. The algebra $G^{s \delta}\left(\mathbb{R}_{+}\right)$with convolution is isomorphic to $E^{s 1}(\mathbb{C})$ with pointwise multiplication, as a real algebra, via the Laplace transform and the inverse Laplace transform.

Proof. The Laplace transform $L$ is injective on $G^{\delta}(\mathbb{R})$. The injectiveness of $L$ extends to $G^{s \delta}(\mathbb{R})$ by definition. Also, $G^{s \delta}\left(\mathbb{R}_{+}\right)$is mapped onto $E^{s 1}(\mathbb{C})$ by $L$ as an algebra homomorphism, by definition.

Note that, by definition, $G^{s \delta}\left(\mathbb{R}_{+}\right)$contains discontinuous functions such as the shifted Heaviside unit functions.

Lemma 7.1. For $f, g \in G^{s \delta}\left(\mathbb{R}_{+}\right)$and for $\mu, s>0$, we have

$$
\begin{aligned}
& (u(t-\mu) f(t-\mu) * u(t-s) g(t-s))(t) \\
& = \begin{cases}0 & \text { if } t<\mu \text { or } t<s, \\
\int_{s}^{t-u} f(t-\tau-\mu) g(\tau-s) d \tau & \text { if } t>\mu \text { and } t>s .\end{cases}
\end{aligned}
$$

Note that in the second non-trivial case, the integral is just the sub-integral of $(\mathrm{f}(\mathrm{t}-\mu) * \mathrm{~g}(\mathrm{t}-$ $s))(t)=\int_{0}^{t} f(t-\tau-\mu) g(\tau-s) d \tau$ restricted to the sub-interval $[s, t-\mu]$ of $[0, t]$, so that we may call the convolution in the statement the sub-convolution. Therefore, 
Corollary 6. The convolution in $\mathrm{G}^{\mathrm{s} \delta}\left(\mathbb{R}_{+}\right)$generates such sub-convolutions, which generate the algebra.

Remark. However, the convolution product in $\mathrm{G}^{\mathrm{s} \delta}\left(\mathbb{R}_{+}\right)$is somewhat difficult to see what it is, but the corresponding point-wise multiplication in $E^{s^{1}}(\mathbb{C})$ is relatively easy to see what it is. As well known in applications to solving ordinary differential equations with initial values, a problem related to $G^{s \delta}\left(\mathbb{R}_{+}\right)$is relatively easily solved in $E^{s 1}(\mathbb{C})$ via the Laplace transform, and then the last task is to compute the corresponding convolution via the inverse Laplace transform.

\section{More with the weak derivative}

Recall that

Lemma 8.1. We have

$$
\mathrm{L}\left(\delta^{\prime}(\mathrm{t})\right)=\mathrm{s}
$$

in the weak sense, i.e., as a functional or a distribution, so that we may define $\mathrm{L}^{-1}(s)=\delta^{\prime}(\mathrm{t})$ for $\mathrm{t} \in \mathbb{R}$ and $s \in \mathbb{C}$.

Proof. We compute

$$
\begin{aligned}
\mathrm{L}\left(\delta^{\prime}(\mathrm{t})\right) & =\int_{0}^{\infty} e^{-s t} \delta^{\prime}(\mathrm{t}) \mathrm{dt} \\
& =\int_{0}^{\infty}(-1) \frac{\partial}{\partial t} e^{-s t} \cdot \delta(t) d t \quad \text { by definition } \\
& =s \mathrm{~L}(\delta(\mathrm{t}))=s
\end{aligned}
$$

Note that the algebra $G(\mathbb{R})$ is closed under the usual derivative and that $G(\mathbb{R})$ is contained in $C^{\infty}(\mathbb{R})$ the algebra of all infinitely many times differentiable (or smooth) functions on $\mathbb{R}$.

Lemma 8.2. For $f \in G(\mathbb{R})$, we have

$$
\left(f * \delta^{\prime}\right)(t)=f^{\prime}(t)
$$

in the weak sense.

Proof. We compute

$$
\begin{aligned}
\left(f * \delta^{\prime}\right)(t) & =\int_{0}^{t} f(t-\tau) \delta^{\prime}(\tau) d \tau \\
& =\int_{0}^{t}(-1) \frac{\partial}{\partial \tau} f(t-\tau) \cdot \delta(\tau) d \tau=\left(f^{\prime} * \delta\right)(t)=f^{\prime}(t) .
\end{aligned}
$$


We define the $n$-th derivative $\delta^{(n)}$ to be the $n$-times convolution with $\delta$. For $f \in G(\mathbb{R})$, we define $f * \delta^{(n)}$ to be $f^{(n)}$ the $n$-th derivative of $f$. We also define $L\left(\delta^{(n)}\right)$ to be $s^{n}$ for $s \in \mathbb{C}$.

We denote by $D_{A}(\mathbb{R})$ the algebra generated by $A(\mathbb{R})$, the delta function $\delta$, and the set $\left\{\delta^{(n)} \mid n \in \mathbb{Z}, n \geq 1\right\}$, with convolution. We also denote by $R(\mathbb{C})$ the algebra of all rational functions on $\mathbb{C}$ with point-wise multiplication.

Proposition 5. There is an algebra isomorphism by the Laplace transform from $D_{A}(\mathbb{R})$ to $R(\mathbb{C})$.

We denote by $D_{G}(\mathbb{R})$ the algebra defined by replacing $A(\mathbb{R})$ with $G(\mathbb{R})$ in the definition of $D_{A}(\mathbb{R})$. We also denote by $R_{E}(\mathbb{C})$ the algebra generated by $R(\mathbb{C})$ and $E(\mathbb{C})$.

Proposition 6. There is an algebra isomorphism by the Laplace transform from $D_{G}(\mathbb{R})$ to $R_{E}(\mathbb{C})$.

Lemma 8.3. For $f \in G^{s \delta}\left(\mathbb{R}_{+}\right)$and $\mu>0$, we have

$$
\left(f * \delta^{\prime}(t-\mu)\right)(t)=u(t-\mu) f^{\prime}(t)
$$

almost everywhere in the weak sense.

Proof. We compute

$$
\begin{aligned}
\left(f * \delta^{\prime}(t-\mu)\right)(t) & =\int_{0}^{t} f(t-\tau) \delta^{\prime}(\tau-\mu) d \tau \\
& =\int_{0}^{t}(-1) \frac{\partial}{\partial \tau} f(t-\tau) \cdot \delta(\tau-\mu) d \tau=\left(f^{\prime} * \delta(t-\mu)\right)(t) \\
& =u(t-\mu) f^{\prime}(t)
\end{aligned}
$$

for $f \in G^{s \delta}\left(\mathbb{R}_{+}\right)$, where note that $f^{\prime}$ is defined almost everywhere (except some finite points of $\left.\mathbb{R}_{+}\right)$.

We denote by $D_{G^{s} \delta}\left(\mathbb{R}_{+}\right)$the algebra generated by $G^{s \delta}\left(\mathbb{R}_{+}\right)$and the weak derivatives $\delta^{(\mathfrak{n})}(t-\mu)$ for $n$ positive integers and $\mu>0$ reals. We also denote by $R_{E^{s 1}}(\mathbb{C})$ the algebra generated by $R(\mathbb{C})$ and $E^{s 1}(\mathbb{C})$.

Proposition 7. There is an algebra isomorphism by the Laplace transform from $\mathrm{D}_{\mathrm{G}^{s}}\left(\mathbb{R}_{+}\right)$to $R_{E^{s} 1}(\mathbb{C})$.

Received: May 2013. Accepted: May 2013. 


\section{References}

[1] Hiroshi FuKawa, Laplace transformation and ordinary differential equations (in Japanese), Sho-Ko-dou (1995).

[2] M. S. J., Mathematics Dictionary, (Sugaku Jiten, in Japanese), Math. Soc. Japan, 4th edition, Iwanami (2007).

[3] T. Sudo, Computing the inverse Laplace transform for rational functions vanishing at infinity, Cubo A Math. J., 16, No 3 (2014), 97-117.

[4] A. VRetblad, Fourier Analysis and Its Applications, GTM 223, Springer (2003). 